in which positions are presented, and their efforts to build coalitions in order to achieve specific policy outcomes. It finds that, while commercial stakeholders support e-cigarette regulation in general (e.g. age restrictions); there are efforts to influence regulation in a way that fits within their economic interests. This project shows that commercial stakeholders seek endorsement from public health organisations, in order to make health claims that can support the 'harm reduction argument'. The presentation will also discuss noncommercial stakeholders' arguments about whether commercial stakeholders should be included in e-cigarette policy debates or not.

\section{P18 NEWLY AT RISK? USING HEALTH SURVEY FOR ENGLAND DATA TO RETROSPECTIVELY EXPLORE THE CHARACTERISTICS OF NEWLY DEFINED AT-RISK DRINKERS FOLLOWING THE CHANGE TO THE UK LOWER RISK DRINKING GUIDELINES}

PC Case* , N Shelton, L Ng Fat. Epidemiology and Public Health, University College London, London, UK

\subsection{6/jech-2018-SSMabstracts. 144}

Background Alcohol guidelines enable individuals to make informed choices about their alcohol consumption and assist healthcare practitioners to identify and offer support to at-risk drinkers. The UK lower risk drinking guidelines were revised in 2016 and the weekly guideline for men was reduced. This study sought to retrospectively establish 1) the number of additional men in England who have been drinking at increasing risk levels in the past 5 years, and 2) whether this group of newly defined increasing risk male drinkers shared any specific characteristics.

Methods Average weekly alcohol consumption data for men aged $16+$ from the cross-sectional nationally representative Health Survey for England were used and regrouped into: non-drinkers; lower risk drinkers ( $\leq 14$ units per week); newly defined increasing risk drinkers ( $>14$ to $\leq 21$ units pw) and increasing/higher risk drinkers ( $>21$ units pw) in order to 1 ) calculate annual population prevalence estimates for newly defined increasing risk adult male drinkers from 2011-2015 $(n=3487-3790)$ and 2) conduct a multinomial logistic regression analysis to assess which characteristics were significantly associated with men being newly defined increasing risk drinkers (reference category) versus lower risk and increasing/ higher risk drinkers $(n=2982)$. Models were fully-adjusted and included age-group, social class, region, smoking status, marital status, ethnicity and limiting-longstanding illness. Analyses were conducted in Stata 15.

Results Population prevalence estimates of newly defined increasing risk drinkers ranged from $10.2 \%$ of the adult male population in England (2,182,401 men) in 2014 to $11.2 \%$ (2,322,896 men) in 2011. Lower risk drinkers were significantly less likely $(\mathrm{p}<0.05)$ than newly defined increasing risk drinkers to be aged 55-64 (RRR 0.43, 95\% CI 0.21 to 0.87); working in professional or managerial occupations (RRR 0.61, 95\% CI 0.45 to 0.83); living in the North East (RRR 0.47, 95\% CI 0.29 to 0.77 ), North West (RRR 0.56, 95\% CI 0.38 to 0.82), West Midlands (RRR 0.52 , 95\% CI 0.32 to 0.83 ) or South West (RRR 0.57 , 95\% CI 0.36 to 0.91 ); and to be exregular (RRR 0.62, 95\% CI 0.46 to 0.83 ) or current (RRR
0.56, 95\% CI 0.39 to 0.81) cigarette smokers. Increasing/ higher risk drinkers were significantly more likely than newly defined increasing risk drinkers to be ex-regular smokers (RRR 1.42, 95\% CI 1.01 to 1.99 ).

Conclusion Approximately $11 \%$ of adult men would have been reclassified from lower risk to increasing risk drinkers according to the 2016 drinking guidelines. Such an increase in at-risk drinkers could impact clinical services. Newly defined increasing risk drinkers differ from lower risk drinkers on several characteristics but are largely similar to increasing/higher risk drinkers, therefore targeting this group specifically may not be feasible.

\section{P19 DEVELOPING A FRAMEWORK FOR PRIORITY SETTING IN AN INTEGRATED HEALTH AND SOCIAL CARE SETTING}

\begin{abstract}
${ }^{1} \mathrm{M}$ Collins ${ }^{*},{ }^{1} \mathrm{R}$ Baker, ${ }^{1} \mathrm{M}$ Mazzei, ${ }^{2} \mathrm{~A}$ Morton, ${ }^{3} \mathrm{~L}$ Frith, ${ }^{4} \mathrm{~K}$ Syrett, ${ }^{5} \mathrm{P}$ Leak, ${ }^{1} \mathrm{C}$ Donaldson. ${ }^{1}$ Yunus Centre for Social Business and Health, Glasgow Caledonian University, Glasgow, UK; ${ }^{2}$ Department of Management Science, University of Strathclyde, Glasgow, UK; ${ }^{3}$ Institute of Psychology, Health and Society, University of Liverpool, Liverpool, UK; ${ }^{4}$ University of Bristol Law School, University of Bristol, Bristol, UK; ${ }^{5}$ Directorate of Health and Social Care, Scottish Government, Edinburgh, UK
\end{abstract}

\subsection{6/jech-2018-SSMabstracts. 145}

Background There is a move, internationally, towards greater integration of health and social care. Integration, it is argued, should reduce budgetary boundaries and facilitate sharing of resources across health and social care. At local levels, delivery organisations need to alter the balance of care from acute settings to people's own home or similar community environments against a background of increasing austerity. To facilitate this shift, there is a need to use robust processes for allocating resources to make difficult decisions and to create interdisciplinary priority setting frameworks involving economists, ethicists, lawyers and decision scientists. In 2014, the Scottish Government established Health and Social Care Partnerships (HSCPs) to deliver this agenda, creating single commissioners and unifying budgets. This paper presents the early stages of a research project funded by the Chief Scientist Office, part of the Scottish Government Health Directorates with the aim to develop and implement an enhanced, multi-disciplinary framework for priority setting, for use by 4 HSCPs, and assess its impact on decision-making and resource allocation.

Methods To develop the framework, a literature review was conducted and the combined framework presented to a multidisciplinary workshop involving academic colleagues, local and national-level stakeholders to gain feedback to develop it further. Participatory Action Research is being undertaken to explore how the framework functioned within complex settings, and how HSCP participants engaged with the framework, and consider how the framework can be adapted to the institutional setting as well as vice versa. Before and after interviews will be conducted.

Results The framework is underpinned by principles from economics (opportunity cost), decision-analysis (good decisions), ethics (justice) and law (fair procedures). It includes key stages for those undertaking priority setting to follow, including: framing the question, looking at current use of resources, defining options and criteria, evaluating the options and 